\title{
Tác động của các yếu tố nội bộ đến nọ̣ xấu của ngân hàng thương mại
}

\section{Impact of internal factors on non-performing loans of Vietnamese commercial banks}

\author{
Nguyễn Kim Phước ${ }^{1 *}$, Phan Ngọc Thùy Như ${ }^{1}$, Ngô Thành Trung ${ }^{1}$ \\ ${ }^{1}$ Trường Đại học Mở Thành phố Hồ Chí Minh, Việt Nam \\ *Tác giả liên hệ, Email: phuoc.nk@ou.edu.vn
}

THÔNG TIN

DOI: $10.46223 /$ HCMCOUJS. econ.vi.13.2.1613.2018

Ngày nhận: 20/11/2017

Ngày nhận lại: 29/11/2017

Duyệt đăng: 20/12/2017

\section{Tù khóa:}

hồi qui dữ liệu bảng, ngân hàng thương mại, nợ xấu, Việt Nam

Keywords:

commercial banks, Nonperforming Loans (NFLs); panel data regression, Vietnam

\section{TÓM TẮT}

Mục tiêu của nghiên cứu là tìm ra các yếu tố nội bộ tác động đến nợ xấu của ngân hàng thương mại ở Việt Nam. Nghiên cứu đã sử dụng dữ liệu thứ cấp từ các báo cáo tài chính hợp nhất và báo cáo thường niên của 22 ngân hàng thương mại hoạt động trong giai đoạn từ 2006 - 2015 (10 năm). Số quan sát tương ứng là 220. Bằng kỹ thuật phân tích hồi quy dữ liệu bảng, hồi quy biến phụ thuộc là nợ xấu, nghiên cứu đã tìm thấy 6 trong 7 yếu tố nội bộ có ảnh hưởng đến nợ xấu của các ngân hàng. Từ kết quả nghiên cứu thực nghiệm, các hàm ý chính sách cho các nhà quản trị ngân hàng được đưa ra nhằm giảm thiểu nợ xấu, từ đó nâng cao hiệu quả hoạt động, đảm bảo sự lành mạnh và an toàn cho các ngân hàng thương mại ở Việt Nam.

\section{ABSTRACT}

The primary objective of this study was to explore the impact of internal factors on non-performing loans (NFL) of commercial banks in Vietnam. The study used secondary data collected from consolidated financial statements and annual reports of 22 commercial banks during a ten-year period from 2006 to 2015. The use of 220 observations and panel data regression analysis with NFLs as dependent variables in the study helped to point out six out of seven internal factors affecting banks' NFLs. These results of empirical research revealed that bank managers and policymakers should focus on internal factors to improve operational efficiency, lower NFLs and ensure financial health for Vietnamese commercial banks. 


\section{Giới thiệu}

Nợ xấu đã và đang là yếu tố quan trọng gây áp lực lớn đối với hệ thống ngân hàng, nguy cơ rủi ro cho "sức khỏe" nền kinh tế. Theo Chính Phủ (2016), nợ xấu của toàn hệ thống ngân hàng 6 tháng đầu năm vẫn còn ở mức $2,78 \%$. Như vậy, nợ xấu chưa thực sự thuyên giảm. Trong 6 tháng đầu năm 2017, VAMC mới chỉ xử lý được 32.400 tỷ đồng nợ xấu, chiếm 13,4\% trong tổng số nợ xấu đã mua (Chu Thai, 2017). Vì thế, vấn đề xử lý nợ xấu vẫn là hoạt động trọng tâm của Ngân hàng Nhà nước và toàn ngành ngân hàng.

Trong hoạt động của các ngân hàng thương mại Việt Nam (NHTMVN) nói riêng và các NHTM nói chung, hoạt động cho vay vẫn là hoạt động chủ yếu. Do tính chất kinh doanh đặc thù này, các NHTM luôn đối diện với rủi ro vỡ nợ của người đi vay. Điều đó tạo ra vấn đề lớn của ngân hàng là nợ xấu (Greenidge \& Grosvenor, 2010; Upal, 2009). Nợ xấu là nguyên nhân chính dẫn đến tình trạng khủng hoảng của các ngân hàng (Guy \& Lowe, 2011). Theo Podpiera và Weill (2008), các đặc điểm của mỗi ngân hàng như: vốn, quản lý hiệu quả chi phí, đa dạng hoạt động tín dụng, hiệu quả sử dụng vốn, ... có ảnh hưởng đến nợ xấu. Louzis, Vouldis, và Metaxas (2012) cho rằng, bên cạnh các biến số nội tại của ngân hàng, các biến số kinh tế vĩ mô cũng có ảnh hưởng đến nợ xấu của ngân hàng.

Kiểm soát và xử lý nợ xấu là một vấn đề trọng tâm của hệ thống ngân hàng. Có nhiều nguyên nhân dẫn đến tình trạng nợ xấu tại các ngân hàng, bao gồm cả nguyên nhân khách quan lẫn nguyên nhân chủ quan. Mục tiêu của nghiên cứu là xem xét các yếu tố nội tại (bên trong) của ngân hàng tác động đến tỷ lệ nợ xấu của hệ thống NHTMVN.

Nội dung nghiên cứu gồm các phần như: Cơ sở lý luận, mô hình, dữ liệu và phương pháp nghiên cứu. Phần cuối cùng là kết quả nghiên cứu và đề xuất hàm ý chính sách cho nhà quản trị.

\section{Cơ sở lý thuyết}

\subsection{Các khái niệm}

Theo International Monetary Fund (2004), một khoản vay được coi là nợ xấu khi quá hạn thanh toán gốc hoặc lãi 90 ngày hoặc hơn; khi các khoản lãi suất đã quá hạn 90 ngày hoặc hơn đã được vốn hóa, cơ cấu lại, hoặc trì hoãn theo thỏa thuận; khi các khoản thanh toán đến hạn dưới 90 ngày nhưng có thể nhận thấy các dấu hiệu rõ ràng cho thấy người vay sẽ không thể hoàn trả nợ đầy đủ.

Định nghĩa nợ xấu theo tiêu chuẩn của Việt Nam được NHNN quy định tại Quyết định số 493/2005/QĐ-NHNN ngày 22/04/2005 và theo Thông tư số $02 / 2013 / T T-N H N N$ ngày 21/01/2013 của Ngân hàng Nhà nước thì nợ xấu là các khoản nợ đã quá hạn thanh toán cả gốc lẫn lãi từ 90 ngày trở lên và khả năng trả nợ là đáng lo ngại. Tỷ lệ nợ xấu/tỷ lệ nợ quá hạn trên 90 ngày $=$ dư nợ quá hạn trên 90 ngày/tổng dư nợ cho vay*100\%.

Theo các khái niệm trên, nợ xấu là nợ quá thời hạn thanh toán cả gốc, lãi từ 90 ngày trở lên. Nghiên cứu này áp dụng số liệu nợ xấu theo khái niệm này vì đây là căn cứ để các ngân hàng thương mại Việt Nam xác định nợ xấu. 
Theo Pham (2005), các nhân tố bên trong nội bộ của chính các NHTM như các nhân tố về năng lực tài chính (ROA, ROE, ROS, ...), khả năng quản trị điều hành, ứng dụng tiến bộ công nghệ, trình độ và chất lượng của lao động, ... có ảnh hưởng đến hiệu quả hoạt động của các ngân hàng.

\subsection{Lý thuyết nền}

Theo lý thuyết rủi ro và quản trị rủi ro cho rằng, hoạt động của các ngân hàng luôn đối diện với các rủi ro tín dụng, rủi ro lãi suất và rủi ro tỷ giá (K. M. Nguyen, 2015). Rủi ro là một tình trạng bất ổn, là sự sai biệt giữa thực tế và kỳ vọng. Trong nghiên cứu này, hai loại rủi ro được xem xét là rủi ro về tín dụng và về lãi suất, vì đây là hai loại rủi ro có liên quan trực tiếp đến nợ xấu của các ngân hàng. Rủi ro tín dụng là rủi ro phát sinh trong quá trình cấp tín dụng của ngân hàng, do người vay suy giảm khả năng trả nợ. Đây là rủi ro thường xuyên và là rủi ro lớn nhất của ngân hàng, nó gây ra nợ xấu của các ngân hàng (Tran, 2011). Nguyên nhân phát sinh rủi ro tín dụng có thể từ phía ngân hàng và từ phía khách hàng. Rủi ro lãi suất là rủi ro do biến động lãi suất gây ra (K. M. Nguyen, 2015).

Mô hình điểm số $Z$ của Atlman (2000), chỉ số $Z$ dùng để đo lường rủi ro tín dụng bao gồm: vốn lưu động/tổng tài sản, lợi nhuận giữ lại/tổng tài sản, EBIT/tổng tài sản, giá trị thị trường của vốn chủ sỡ hữu/giá trị sổ sách của tổng nợ và doanh số/tổng tại sản (Tran, 2011). Trị số $\mathrm{Z}$ càng cao thì xác suất vỡ nợ của người đi vay càng thấp. Hay nói khác hơn là nợ xấu của các ngân hàng càng thấp.

Lý thuyết thông tin bất cân xứng giải thích rằng, bên nào có nhiều thông tin hơn về giao dịch thì bên đó có lợi thế hơn bên kia do thông tin có ảnh hưởng đến việc ra quyết định (Auronen, 2003; Richard, 2011). Điều này dẫn đến những lựa chọn bất lợi, tích tụ các khoản nợ xấu tại ngân hàng (Bester, 1994).

\subsection{Các nghiên cúu trước có liên quan}

Kết quả nghiên cứu của Sinkey và Greenwalt (1991) đã chứng minh, việc mở rộng tín dụng cũng là một nguyên nhân gây ra nợ xấu cao, do việc kiểm tra và lựa chọn người vay kém chất lượng. Kết quả này cũng được kiểm chứng lại qua nghiên cứu của Jiménez và Saurian (2006). Golden và Walker (1993) qua kết quả nghiên cứu của mình đã kết luận rằng, thông tin tín dụng có ảnh hưởng đến việc ra quyết định cho vay của ngân hàng. Nghĩa là, nếu ngân hàng có nhiều thông tin hơn về khách hàng, chất lượng thông tin tốt sẽ giúp giảm rủi ro tín dụng, từ đó giảm các khoản nợ xấu của ngân hàng.

Salas và Saurina (2002) nghiên cứu yếu tố quyết định cho vay của ngân hàng thương mại Tây Ban Nha, bằng cách sử dụng một mô hình dữ liệu bảng trong giai đoạn 1985-1997. Phát hiện của nghiên cứu này là sự thay đổi trong nợ xấu của các ngân hàng được giải thích bởi các yếu tố như: Tăng trưởng GDP, mở rộng tín dụng, kích thước ngân hàng, tỷ lệ vốn và sức mạnh thị trường. Trong quá trình nghiên cứu các yếu tố tác động đến tỷ lệ nợ xấu của các NHTM ở châu Âu, Salas và Saurina (2002) cũng đã phát hiện tốc độ tăng trưởng tín dụng (credit growth) và nợ xấu có mối tương quan thuận.

Nghiên cứu về các yếu tố ảnh hưởng đến tỷ lệ nợ xấu của hệ thống ngân hàng tại 16 quốc gia thuộc vùng cận Sahara từ năm 1993-2002, Fofack (2005) đã chứng minh rằng, ROE 
có tác động trái chiều đến nợ xấu. Mario (2006) nghiên cứu về rủi ro của hệ thống ngân hàng ở Italia trong giai đoạn từ 1985-2002, đã chỉ ra mối tương quan thuận giữa nợ xấu với tỷ lệ dự phòng rủi ro tín dụng.

Đánh giá về nợ xấu tại Ấn Độ thì Dash và Kabra (2010) thấy rằng lãi suất thực càng tăng thì nợ xấu càng tăng, còn quy mô ngân hàng (size) lại không có tác động đến nợ xấu. Cũng từ bộ dữ liệu bảng của các ngân hàng Ấn Độ trong giai đoạn 1998 - 2008, các tác giả còn thấy tốc độ tăng trưởng tín dụng (credit growth) và nợ xấu có tương quan nghịch.

Louzis, Vouldis, và Metaxas (2010) nghiên cứu nợ xấu theo các khoản vay của hệ thống ngân hàng ở Hy lạp đã chỉ ra rằng, lãi suất cho vay có tác động cùng chiều với tỷ lệ nợ xấu của các ngân hàng. Các tác giả cũng chỉ ra rằng có thể nhìn vào hiệu quả hoạt động của ngân hàng, thông qua ROA và ROE, để đánh giá xu hướng nợ xấu trong tương lai vì chúng có tương quan nghịch với nợ xấu.

Messai và Jouini (2013) nghiên cứu các yếu tố quyết định cho các khoản nợ xấu cho một mẫu 85 ngân hàng ở ba nước (Ý, Hy Lạp và Tây Ban Nha) trong giai đoạn 2004-2008. Phương pháp hồi quy dữ liệu bảng, tác giả đã phát hiện ra khoản nợ xấu có quan hệ nghịch với khoản dự phòng rủi ro mất vốn và lãi suất thực.

V. H. T. Nguyen (2015) phân tích yếu tố tác động đến nợ xấu các NHTMVN, tác giả sử dụng mô hình bao gồm các biến vĩ mô và vi mô, trong đó, các yếu tố liên quan đến ngân hàng có ROE, tổng tài sản, tăng trưởng tín dụng, qui mô ngân hàng. Tác giả sử dụng hồi quy $\mathrm{FE}, \mathrm{RE}$ và $D G M M$ để kiểm định các yếu tố có tác động đến nợ xấu với bảng dữ liệu từ năm 2007 2014. Kết quả nghiên cứu cho thấy, ROE, lạm phát và tăng trưởng kinh tế có tác động trái chiều đến nợ xấu, qui mô ngân hàng, tăng trưởng tín dụng, vốn chủ sở hữu/tổng tài sản có tác động cùng chiều với nợ xấu.

\section{Mô hình, dữ liệu và phương pháp nghiên cứu}

Trên cơ sở lý thuyết nền và các nghiên cứu trước có liên quan đến nghiên cứu về nợ xấu tại các ngân hàng thương mại, mô hình nghiên cứu được đề xuất.

\section{Bảng 1}

Cơ sở chọn biến trong mô hình nghiên cứu

\begin{tabular}{|l|l|l|l|l|}
\hline \multicolumn{1}{|c|}{ Tên biến } & \multicolumn{1}{|c|}{ Mô tả } & \multicolumn{1}{|c|}{ Công thức } & $\begin{array}{c}\text { Dấu tác } \\
\text { dộng }\end{array}$ & \multicolumn{1}{|c|}{ Nghiên cứu trước } \\
\hline ROE & $\begin{array}{l}\text { Suất sinh lợi } \\
\text { trên vốn chủ } \\
\text { sở hữu }\end{array}$ & $\begin{array}{l}\text { Lợi nhuấn sau } \\
\text { thuế/vốn chủ sở hữu } \\
(\%)\end{array}$ & - & $\begin{array}{l}\text { Fofack (2005); Louzis } \\
\text { và cộng sự (2010) }\end{array}$ \\
\hline LnCRE & $\begin{array}{l}\text { Tăng trưởng } \\
\text { tín dụng }\end{array}$ & $\begin{array}{l}\text { Ln tăng trưởng tín } \\
\text { dụng của ngân hàng }\end{array}$ & + & $\begin{array}{l}\text { Salas và Saurina } \\
\text { (2002); Jiménez và } \\
\text { Saurian (2006); V. H. } \\
\text { T. Nguyen (2015) }\end{array}$ \\
\hline LnRISK & $\begin{array}{l}\text { Dự phòng rủi } \\
\text { ro }\end{array}$ & $\begin{array}{l}\text { Ln dự phòng rủi ro } \\
\text { tín dụng }\end{array}$ & + & Mario (2006) \\
\hline
\end{tabular}




\begin{tabular}{|l|l|l|c|l|}
\hline \multicolumn{1}{|c|}{ Tên biến } & \multicolumn{1}{|c|}{ Mô tả } & \multicolumn{1}{|c|}{ Công thức } & $\begin{array}{c}\text { Dấu tác } \\
\text { dộng }\end{array}$ & \multicolumn{1}{|c|}{ Nghiên cứu trước } \\
\hline LnASSETS & Tổng tài sản & $\begin{array}{l}\text { Ln tông tài sản của } \\
\text { ngân hàng }\end{array}$ & - & $\begin{array}{l}\text { Louzis và cộng sự } \\
(2010)\end{array}$ \\
\hline AGENT_branch & $\begin{array}{l}\text { Nhân viên/chi } \\
\text { nhánh }\end{array}$ & $\begin{array}{l}\text { Số nhân viên bình } \\
\text { quân/chi nhánh } \\
\text { (người/chi nhánh) }\end{array}$ & + & $\begin{array}{l}\text { Salas và Saurina } \\
(2002) ; \text { V. H. T. } \\
\text { Nguyen (2015) }\end{array}$ \\
\hline INTEREST & $\begin{array}{l}\text { Lãi suất tái } \\
\text { cấp vốn }\end{array}$ & $\begin{array}{l}\text { Lãi suất tái cấp vốn } \\
(\%)\end{array}$ & + & $\begin{array}{l}\text { Dash và Kabra } \\
(2010) ; \text { Louzis và } \\
\text { cộng sự (2010) }\end{array}$ \\
\hline ICT & $\begin{array}{l}\text { Úng dụng } \\
\text { công nghệ } \\
\text { trong ngân } \\
\text { hàng }\end{array}$ & $\begin{array}{l}\text { Chî̉ số ứng dụng } \\
\text { công nghệ trong } \\
\text { ngân hàng }\end{array}$ & $\begin{array}{l}\text { Golden và Walker } \\
(1993) ; \text { Auronen } \\
\text { (2003); Harper } \\
(2011)\end{array}$ \\
\hline
\end{tabular}

Nguồn: Kết quả phân tích dữ liệu của nhóm nghiên cứu

Qua lược khảo các nghiên cứu trước, tác giả nhận thấy có rất nhiều yếu tố ảnh hưởng đến tỷ lệ nợ xấu tại ngân hàng thương mại. Dựa trên ý tưởng và cách tiếp cận vấn đề trong nghiên cứu của Golden và Walker (1993); Salas và Saurina (2002); Auronen (2003); Mario (2006); Louzis và cộng sự (2010), Richard (2011), Harper (2011) và V. H. T. Nguyen (2015) làm nền tảng, nghiên cứu này tiếp cận ở khía cạnh sự tác động của các yếu tố nội bộ tác động đến nợ xấu (NPL) của các NHTMVN giai đoạn 2006-2015.

\subsection{Mô hình nghiên cúu}

Mô hình nghiên cứu được đề xuất dựa theo cơ sở lý luận và đặc thù của các ngân hàng thương mại Việt Nam, cũng như tính phù hợp của dữ liệu thu thập được. Mô hình nghiên cứu có dạng như sau:

$$
\pi_{\mathrm{it}}=\mathrm{c}_{\mathrm{it}}+\alpha_{\mathrm{j}} \mathrm{X}_{\mathrm{it}}+\varepsilon_{\mathrm{it}}
$$

Trong đó, $\pi$ là biến phụ thuộc được đo lường bằng tỷ số NPL, c là hệ số chặn, $\alpha$ là hệ số hồi quy, $\mathrm{X}$ đại diện cho các biến đặc trưng bên trong ngân hàng. Kí hiệu i đại diện cho thứ tự các ngân hàng, $t$ là năm. Mô hình nghiên cứu chi tiết như sau:

$$
\begin{aligned}
& \mathrm{NPL}_{\mathrm{it}}=c_{\mathrm{it}}+\alpha_{1} \mathrm{ROE}_{\mathrm{it}}+\alpha_{2} \mathrm{LnCRE}_{\mathrm{it}}+\alpha_{3} \operatorname{LnRISK}_{\mathrm{it}}+\alpha_{4} \text { LnASSETS }_{\mathrm{it}} \\
& +\alpha_{5} \text { AGENT_branch }_{\text {it }}+\alpha_{6} \text { INTEREST }_{\text {it }}+\alpha_{7} \mathrm{ICT}_{\text {it }}+\varepsilon_{\mathrm{it}}
\end{aligned}
$$

NPL: Non - performing loan (\%) - biến phụ thuộc. Tỷ lệ nợ xấu càng cao chứng tỏ khả năng sử dụng vốn không hiệu quả và làm giảm lợi nhuận của ngân hàng. Tỷ lệ nợ xấu của các ngân hàng được nêu trong báo cáo tài chính (đã kiểm toán) hằng năm.

ROE: Suất sinh lời trên vốn chủ sở hữu (\%). Biến định lượng này biểu thị cho khả năng sử dụng vốn hiệu quả của hệ thống ngân hàng. $\mathrm{ROE}$ có quan hệ nghịch biến với nợ xấu (kỳ vọng dấu âm). Nghiên cứu của Louzis và cộng sự $(2010,2012)$ đã tìm thấy mối quan hệ trái chiều giữa kết quả kinh doanh và nợ xấu. $\mathrm{ROE}$ của các ngân hàng càng tăng cho thấy hoạt động kinh doanh của các ngân hàng mang lại hiệu quả cao thì tỷ lệ nợ xấu thấp. 
LnCRE: Tăng trưởng tín dụng. Biến này thể hiện quy mô vốn được cung cấp ra thị trường. Tăng trưởng tín dụng cao góp phần làm giảm tỷ lệ nợ xấu của các ngân hàng ở năm hiện tại (Dash \& Kabra, 2010). Tuy nhiên, tốc độ tăng trưởng tín dụng cao trong quá khứ sẽ góp phần làm cho nợ xấu ở năm hiện tại tăng lên, kết quả này cũng phù hợp với tình hình hoạt động của hệ thống NHTMVN, nghĩa là tăng trưởng tín dụng có quan hệ đồng biến (kỳ vọng dấu +) với nợ xấu (Mario, 2006).

LnRISK: Lượng dự phòng rủi ro tín dụng thể hiện lượng vốn bị giữ lại không đưa vào thị trường. Dự phòng rủi ro là số tiền được trích lập và hạch toán vào chi phí hoạt động để dự phòng cho những tổn thất có thể xảy ra đối với các khoản cho vay của ngân hàng thương mại. Ngân hàng dự phòng càng nhiều thể hiện mức độ rủi ro càng cao. Mario (2006) cho rằng, các ngân hàng trích lập dự phòng theo đánh giá mức độ rủi ro của các khoản tín dụng, nếu rủi ro càng cao thì trích lập dự phòng càng lớn (kỳ vọng dấu +).

LnASSETS: Nghiên cứu của Louzis và cộng sự (2010) cho thấy quy mô ngân hàng tác động trái chiều lên tỷ lệ nợ xấu tại ngân hàng. Đối với một ngân hàng, tổng tài sản thường là một con số có giá trị tuyệt đối rất lớn, do đó trong kỹ thuật hồi quy lúc phân tích dữ liệu, các tác giả thường lấy logarit (tổng tài sản) làm biến đại diện cho quy mô. Trong đó khoản mục tổng tài sản thể hiện trên bảng cân đối kế toán hàng năm của các ngân hàng. Biến này được kỳ vọng có quan hệ nghịch biến với nợ xấu, nghĩa là tổng tài sản của ngân hàng càng tăng thì tỷ lệ nợ xấu càng giảm (-).

AGENT_branch: Số lượng nhân viên bình quân/điểm giao dịch của từng ngân hàng qua các năm. Biến này cũng được chọn là một biến đại diện cho qui mô và hiệu quả làm việc của các ngân hàng. Theo Salas và Saurina (2002) và V. H. T. Nguyen (2015) qui mô ngân hàng càng lớn (tài sản, chi nhánh, lực lượng lao động) thì việc kiểm soát nợ xấu càng khó, do các ngân hàng còn có hạn chế về nhiều vấn đề trong đó có năng lực quản lý con người và rủi ro đạo đức. Biến này kỳ vọng mang dấu dương $(+)$ nghĩa là AGENT_branch càng lớn thì NPL càng tăng.

INTEREST: Lãi suất tái cấp vốn (\%). Biến này thể hiện nhu cầu vốn trong nền kinh tế. Lãi suất tái cấp vốn của NHNN cho NHTM, xem như là mức lãi mà NHTM dựa vào đó để ân định mức lãi suất cho vay phù hợp. Theo Dash và Kabra (2010); Louzis và cộng sự (2010) lãi suất có tác động cùng chiều với nợ xấu bởi vì khi lãi suất càng cao thì khả năng trả nợ của người vay càng giảm hay nói cách khác là, khả năng thu hồi vốn và lãi đúng hạn của ngân hàng giảm, nợ xấu tăng (kỳ vọng dấu dương $(+)$ ).

ICT (Việt Nam ICT Index): Chỉ số ứng dụng công nghệ trong ngân hàng được trích dữ liệu của Bộ Thông tin và Truyền thông kết hợp Hội tin học Việt Nam. Chỉ số càng cao càng thể hiện khả năng tiếp cận và ứng dụng công nghệ càng cao, qua đó góp phần trong kiểm soát rủi ro trong cho vay, giảm thiểu khả năng mất vốn. Theo các nghiên cứu của Golden và Walker (1993); Auronen (2003) và Harper (2011), thông tin càng rõ ràng, chính xác, đầy đủ, không có trường hợp thông tin bất cân xứng thì càng giúp ngân hàng giảm tỷ lệ nợ xấu nhờ quyết định cung cấp tín dụng chính xác (kỳ vọng dấu âm (-)). 


\subsection{Dũ liệu và phương pháp nghiên cứu}

Mẫu nghiên cứu bao gồm 22 ngân hàng với tổng cộng 220 quan sát theo năm cho dữ liệu bảng cân bằng trong 10 năm từ năm 2006 đến năm 2015. Dữ liệu nghiên cứu được thu thập từ các báo cáo tài chính của các NHTMVN từ năm 2006 - 2015. Riêng chỉ số thông tin tín dụng được thu thập từ Bộ Thông tin và Truyền thông kết hợp Hội tin học Việt Nam (https://cic.org.vn).

Kỹ thuật hồi quy bảng được sử dụng để phân tích tác động của các biến đặc trưng bên trong ngân hàng đối với NPL. Trong nghiên cứu này, do số lượng các đơn vị chéo $(\mathrm{n}=20)$ lớn hơn nhiều so với chuỗi thời gian $(\mathrm{t}=10)$ nên tác giả quan tâm đến tác động của các đơn vị chéo hay sự khác biệt về điều kiện đặc thù của các đơn vị chéo có ý nghĩa như thế nào đối với biến phụ thuộc (NPL). Vì vậy, tác giả sẽ lần lượt thực hiện các mô hình hồi quy như mô hình Pooled (hồi quy OLS gộp -Pooled), mô hình tác động cố định $(F E)$ và mô hình tác động ngẫu nhiên $(R E)$ của các đơn vị chéo, từ đó chọn ra mô hình phù hợp.

Kiểm định Wald nhằm mục đích xác định hệ số tung độ gốc của các đơn vị chéo (20 ngân hàng) có bằng nhau hay không, nếu bằng nhau tức là thỏa trường hợp hệ số trục tung và hệ số độ dốc không thay đổi, hay mô hình Pooled là phù hợp.

Kiểm định Hausman được thực hiện để lựa chọn giữa mô hình FE và RE với giả thuyết $\mathrm{H}_{0}$ là không có sự khác biệt giữa phương pháp $\mathrm{FE}$ và $\mathrm{RE}$ (hay không có sự tương quan giữa biến độc lập và yếu tố ngẫu nhiên $u_{i}$ vì tương quan là nguyên nhân tạo nên sự khác biệt giữa $F E$ và $R E$ ). Nếu giá trị Prob của kiểm định Hausman $\leq \alpha=5 \%$ thì bác bỏ giả thiết $\mathrm{H}_{0}$ tức mô hình FE phù hợp, ngược lại thì mô hình RE sẽ được lựa chọn.

Kiểm định sự phù hợp của các hệ số hồi quy nhằm xem xét mối quan hệ tuyến tính giữa các biến độc lập với biến phụ thuộc. Mô hình được xem là không phù hợp khi tất cả các hệ số hồi quy bằng không và mô hình được xem là phù hợp khi có ít nhất một hệ số hồi quy khác không.

Kiểm định phương sai sai số thay đổi theo phương pháp Breusch và Pagan (1979). Dựa vào chỉ số Prob của giá trị kiểm định Chi-square để quyết định chấp nhận hay bác bỏ giả thuyết $\mathrm{H}_{0}$. Nếu Prob $>\alpha=5 \%$ thì không bác bỏ giả thuyết $\mathrm{H}_{0}$, tức mô hình không xảy ra tương quan sai số thay đổi.

Kiểm định tương quan chuỗi: Theo Wooldridge (2002), chúng ta có thể kiểm định tương quan chuỗi bậc 1 bằng cách hồi quy phần dư thu được ở mô hình gốc với biến trễ của nó như mô hình $\varepsilon_{\mathrm{t}}=\rho \varepsilon_{(\mathrm{t}-1)}+\mathrm{u}_{\mathrm{t}}$ và sau đó tiến hành kiểm định Wald cho mô hình này.

Nếu xảy ra tương quan chuỗi bậc 1 thì hệ số $\rho$ sẽ nhận giá trị là $-0,5$. Do đó, giả thuyết $\mathrm{H}_{0}$ của kiểm định Wald chính là $\rho=-0,5$, tức là có xảy ra tương quan chuỗi bậc 1 . Nếu giá trị p-value $\leq$ mức ý nghĩa $\alpha$ thì ta bác bỏ giả thuyết $\mathrm{H}_{0}$, có nghĩa hiện tượng tương quan chuỗi không xảy ra và ngược lại.

Trong trường hợp khi thực hiện hồi quy mô hình, ở bước kiểm định phần dư của mô hình, nếu xảy ra hiện tượng tự tương quan phần dư hay phương sai sai số thay đổi có khả năng làm sai lệch kết quả, nghiên cứu sẽ tiếp tục xử lý bằng cách sử dụng phương pháp hồi quy GLS (Generalized Least Square - hàm ước lượng bình phương tối thiểu tổng quát) để cho ra kết quả 
hồi quy chính xác hơn. Uớc lượng GLS khả thi (FGLS) dùng để khắc phục hiện tượng phương sai sai số và tự tương quan (Gujarati, 2004, p. 641). GLS cho phép ước lượng với sự có mặt của độ tương quan $\mathrm{AR}(1)$ trong các bảng và mối tương quan chéo, tính không đều của dữ liệu bảng. Khi hồi quy Pooled (OLS) có hiện tượng phương sai sai số và tự tương quan nghĩa là mô hình có khuyết tật, phương pháp GLS khả thi (FGLS) bằng cách dùng biến đổi tương đương để đưa ra một mô hình mới mà sai số ngẫu nhiên trong mô hình này có phương sai sai số không đổi, sau đó dùng Pooled (OLS) để ước lượng mô hình mới này (Baltagi, 2010).

\section{Phân tích kết quả nghiên cứu}

\subsection{Phân tích thống kê mô tả}

Dữ liệu nghiên cứu gồm 220 quan sát (10 năm với 22 ngân hàng). Sau khi kiểm tra tính đầy đủ và chính xác của dữ liệu, nghiên cứu thực hiện thống kê mô tả nhằm cung cấp những thông tin cơ bản và tổng quan về tình hình hoạt động kinh doanh của các NHTMVN. Kết quả thống kê mô tả được thể hiện chi tiết theo Bảng 2.

\section{Bảng 2}

Thống kê mô tả giá trị các biến trong mô hình

\begin{tabular}{|c|c|c|c|c|c|c|c|c|}
\hline BANK & NPL & ROE & CRE & RISK & ASSETS & $\begin{array}{c}\text { AGENT } \\
\text { branch }\end{array}$ & INTEREST & ICT \\
\hline ABB & 3.04 & 5.85 & 16649.04 & 274.98 & 37567.56 & 19.65 & 6.458 & 0.5844 \\
\hline ACB & 1.18 & 23.01 & 79158.88 & 866.89 & 160731.70 & 31.34 & 6.458 & 0.5781 \\
\hline BIDV & 3.38 & 14.42 & 295569.20 & 6797.26 & 419387.40 & 25.64 & 6.458 & 0.8082 \\
\hline CTG & 1.05 & 17.28 & 268219.80 & 2714.66 & 408768.10 & 18.24 & 6.458 & 0.6620 \\
\hline EIB & 1.89 & 10.52 & 55546.55 & 533.25 & 110538.90 & 31.15 & 6.458 & 0.5496 \\
\hline HDB & 1.46 & 10.91 & 21530.19 & 246.62 & 47095.69 & 24.84 & 6.458 & 0.4746 \\
\hline KLB & 1.84 & 8.55 & 22195.43 & 75.13 & 13230.16 & 28.41 & 6.458 & 0.3852 \\
\hline MB & 1.91 & 18.17 & 55476.45 & 1028.40 & 118248.40 & 27.58 & 6.458 & 0.6514 \\
\hline MSB & 2.23 & 9.70 & 22203.36 & 360.21 & 77802.61 & 19.02 & 6.458 & 0.5600 \\
\hline NAB & 1.90 & 5.87 & 8090.08 & 65.86 & 17690.60 & 19.48 & 6.458 & 0.4782 \\
\hline NVB & 2.87 & 7.00 & 10726.62 & 124.34 & 21886.42 & 15.55 & 6.458 & 0.6250 \\
\hline OCB & 2.45 & 11.36 & 14303.83 & 158.31 & 23484.87 & 21.52 & 6.458 & 0.6177 \\
\hline PGB & 2.39 & 9.13 & 9239.42 & 121.52 & 15101.88 & 20.19 & 6.458 & 0.5006 \\
\hline SCB & 3.39 & 9.97 & 66314.66 & 666.43 & 117896.40 & 17.39 & 6.458 & 0.5425 \\
\hline SEA & 1.86 & 5.44 & 18426.41 & 278.05 & 124121.60 & 15.35 & 6.458 & 0.4955 \\
\hline SHB & 2.83 & 8.58 & 44626.77 & 569.64 & 81144.61 & 14.78 & 6.458 & 0.4975 \\
\hline STB & 0.99 & 11.25 & 71048.03 & 821.55 & 132651.20 & 25.25 & 6.458 & 0.5263 \\
\hline TCB & 2.50 & 18.10 & 50388.33 & 4863.20 & 124105.00 & 24.30 & 6.458 & 0.5178 \\
\hline VAB & 2.09 & 7.01 & 11540.50 & 140.84 & 21544.86 & 21.77 & 6.458 & 0.5376 \\
\hline VCB & 2.78 & 18.68 & 201494.80 & 5122.29 & 364296.20 & 35.11 & 6.458 & $\mathbf{0 . 6 1 9 8}$ \\
\hline VIB & 2.01 & 9.15 & 31420.31 & 489.40 & 63839.97 & 24.66 & 6.458 & 0.5466 \\
\hline VPB & 1.92 & 13.37 & 36313.35 & 406.94 & 77501.13 & 28.44 & 6.458 & 0.4487 \\
\hline Total & 2.18 & 11.51 & 64112.82 & 1214.81 & 117210.70 & 23.17 & 6.458 & 0.5549 \\
\hline
\end{tabular}

Nguồn: Tác giả (2017) 
Kết quả mô tả các giá trị thống kê (Bảng 2) các biến cho thấy: Tỷ lệ nợ xấu (NPL) của các ngân hàng giai đoạn từ năm 2006 đến 2015 ở mức trung bình là $2.18 \%$. Ngân hàng có tỷ lệ nợ xấu nhỏ nhất là STB với $0.99 \%$ và ngân hàng có tỷ lệ nợ xấu cao nhất là $\mathrm{SCB}$ là $3.39 \%$. Các ngân hàng $\mathrm{BIDV}, \mathrm{VCB}, \mathrm{CTG}$ là những ngân hàng có tăng trưởng tín dụng cao nhất và cũng là nhóm các ngân hàng có chỉ số ứng dụng thông tin tín dụng (ICT) cao. Xét trên qui mô tổng tài sản, hai ngân hàng $\mathrm{KLB}, \mathrm{PGB}$ có giá trị tổng tài sản nhỏ nhất. Các ngân hàng $\mathrm{ABB}, \mathrm{NAB}$ và SEA có hệ số ROE rất thấp, chưa đến $1 \frac{1}{2}$ bình quân toàn ngành ngân hàng trong cả giai đoạn 2006 - 2015. Về lãi suất tái cấp vốn trong giai đoạn 2006 - 2015, biến động lãi suất khá mạnh, đỉnh điểm là năm 2011. Những năm sau 2011, lãi suất biến động theo chiều hướng giảm dần và ổn định ở mức khoảng $5 \% / n a ̆ m$. Đây là mức lãi suất vừa phải, phù hợp với lợi nhuận bình quân của ngành ngân hàng, lạm phát và tăng trưởng kinh tế của Việt Nam giai đoạn hiện nay.

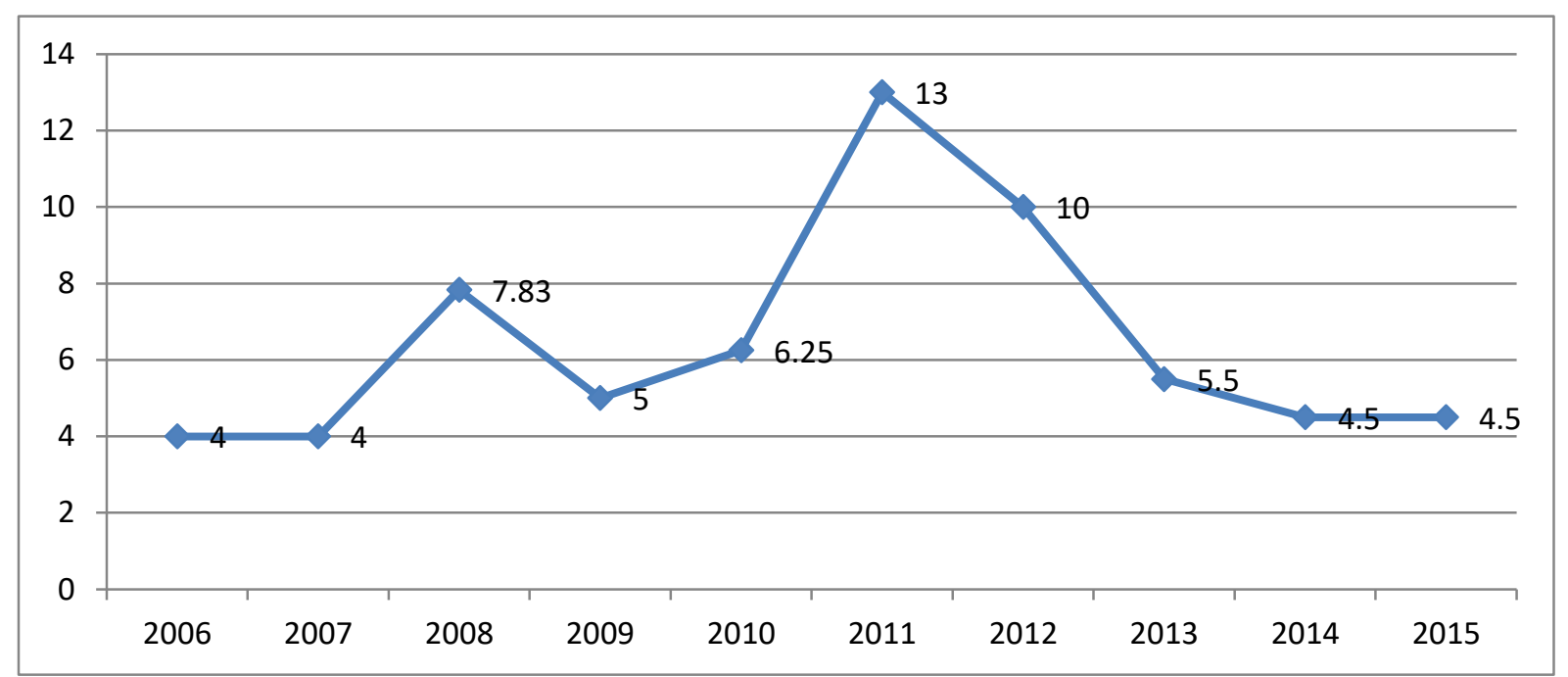

Hình 1. Biến động lãi suất tái cấp vốn giai đoạn 2006 - 2015

Nguồn: Tác giả (2017)

\subsection{Phân tích hồi quy}

Kết quả phân tích hồi quy biến phụ thuộc (tỷ lệ nợ xấu (\%) - NPL) theo các biến độc lập bên trong bằng phương pháp ước lượng OLS dữ liệu gộp (Pooled), phương pháp tác động cố định (FE) và tác động ngẫu nhiên (RE) của các đơn vị chéo được thể hiện trong Bảng 3 dưới đây:

\section{Bảng 3}

Kết quả phân tích hồi quy các biến tác động đến tỷ lệ nợ xấu (\%) - NPL

\begin{tabular}{|c|c|c|c|c|c|c|}
\hline \multirow{3}{*}{$\begin{array}{l}\text { INDEPENDENT } \\
\text { VARIABLES }\end{array}$} & \multicolumn{6}{|c|}{ MODEL REGRESSION } \\
\hline & \multicolumn{2}{|c|}{ POOLED } & \multicolumn{2}{|c|}{ FE } & \multicolumn{2}{|c|}{ RE } \\
\hline & Coef. & $\begin{array}{l}\text { Sta. } \\
\text { Err. }\end{array}$ & Coef. & $\begin{array}{l}\text { Sta. } \\
\text { Err. }\end{array}$ & Coef. & $\begin{array}{l}\text { Sta. } \\
\text { Err. }\end{array}$ \\
\hline LnASSETS & $-0.944 * * *$ & -4.39 & $-0.973 * * *$ & -3.69 & $-0.945^{* * *}$ & -4.20 \\
\hline LnCRE & -0.221 & -1.21 & -0.170 & -0.83 & -0.205 & -1.10 \\
\hline
\end{tabular}




\begin{tabular}{|c|c|c|c|c|c|c|}
\hline \multirow{3}{*}{$\begin{array}{l}\text { INDEPENDENT } \\
\text { VARIABLES }\end{array}$} & \multicolumn{6}{|c|}{ MODEL REGRESSION } \\
\hline & \multicolumn{2}{|c|}{ POOLED } & \multicolumn{2}{|c|}{ FE } & \multicolumn{2}{|c|}{ RE } \\
\hline & Coef. & $\begin{array}{l}\text { Sta. } \\
\text { Err. }\end{array}$ & Coef. & $\begin{array}{l}\text { Sta. } \\
\text { Err. }\end{array}$ & Coef. & $\begin{array}{l}\text { Sta. } \\
\text { Err. }\end{array}$ \\
\hline LnRISK & $0.996 * * *$ & 7.48 & $0.941 * * *$ & 6.13 & $0.978 * * *$ & 7.16 \\
\hline ROE & $-0.050 * * *$ & -3.51 & $-0.059 * * *$ & -3.37 & $-0.053 * * *$ & -3.57 \\
\hline AGENT_branch & -0.010 & -0.73 & 0.012 & 0.66 & -0.005 & -0.30 \\
\hline INTEREST & 0.053 & 1.55 & $0.067 * *$ & 1.98 & $0.057 *$ & 1.70 \\
\hline ICT & $-1.441 *$ & 1.79 & 0.990 & 0.86 & -1.401 & 1.63 \\
\hline Number of obs & \multicolumn{2}{|c|}{220} & \multicolumn{2}{|c|}{220} & \multicolumn{2}{|c|}{220} \\
\hline Adj R-squared & \multicolumn{2}{|c|}{0.296} & \multicolumn{2}{|c|}{0.280} & \multirow{2}{*}{\multicolumn{2}{|c|}{0.0000}} \\
\hline Prob $>F$ & \multicolumn{2}{|c|}{0.0000} & \multicolumn{2}{|c|}{0.000} & & \\
\hline
\end{tabular}

Mức ý nghĩa: * $\mathrm{P}<0.1, * * \mathrm{P}<0.05$ và $* * * \mathrm{P}<0.01$

Nguồn: Tác giả (2017)

Từ kết quả hồi quy Pooled, FE và RE (bảng 3) ta thấy, các biến ROE, LnRISK và LnASSETS luôn có tác động đến NPL. Mô hình hồi quy đều có ý nghĩa thống kê và có Adj Rsquared từ $28 \%$ trở lên.

Kiểm định Hausman để lựa chọn giữa mô hình $\mathrm{FE}$ và RE. Kết quả kiểm định cho giá trị $\chi^{2} \approx 0$ và $\operatorname{Prob}>\operatorname{chi} 2=0.856>\alpha=0.05$ nên ta bác bỏ giả thuyết $\mathrm{H}_{0}$. Như vậy, mô hình $\mathrm{RE}$ phù hợp hơn mô hình FE. Kiểm định Wald để lựa chọn giữa mô hình Pooled và $\mathrm{FE}$ : Kết quả kiểm định cho giá trị Chi-square $=37.084$ và Prob $\approx 0.1147>\alpha=0.05$ nên ta chấp nhận giả thuyết $\mathrm{H}_{0}$. Mô hình Pooled là phù hợp hơn.

Kiểm định Breusch - Pagan cho mình hình Pooled cho kết quả Prob $>$ Chi $2=0.000<\alpha$ $=0.05$ nên mô hình có xảy ra hiện tượng phương sai sai số thay đổi. Đồng thời kiểm định Wald theo Worldridge cho Prob>F $=0.000<\alpha=0.05$ nên mô hình có xảy ra hiện tượng tự tương quan. Nghiên cứu sử dụng phương pháp FGLS để khắc phục các hiện tượng tương quan chuỗi và phương sai sai số thay đổi. Phân tích hồi quy theo phương pháp FGLS cho kết quả như bảng 4.

\section{Bảng 4}

Kết quả lựa chọn mô hình phù hợp

\begin{tabular}{|l|cc|c|}
\hline \multirow{2}{*}{\multicolumn{1}{c|}{$\begin{array}{c}\text { INDEPENDENT } \\
\text { VARIABLES }\end{array}$}} & \multicolumn{2}{c|}{$\begin{array}{c}\text { MODEL REGRESSION } \\
\text { (FGLS) }\end{array}$} & $\begin{array}{c}\text { Kết quả kiểm định giả } \\
\text { thuyết }\end{array}$ \\
\cline { 2 - 3 } & Coef. & Sta. Err. & \\
\hline LnASSETS & $-0.4696 * * *$ & 0.1467 & Chấp nhận \\
LnCRE & $-0.4289 * * *$ & 0.1331 & Chấp nhận \\
LnRISK & $0.7522 * * *$ & 0.1020 & Chấp nhận \\
ROE & $-0.0388^{*} * *$ & 0.0084 & Chấp nhấn \\
AGENT_branch & 0.0045 & 0.0092 & Từ chối \\
\hline
\end{tabular}




\begin{tabular}{|c|c|c|c|}
\hline \multirow{2}{*}{$\begin{array}{l}\text { INDEPENDENT } \\
\text { VARIABLES }\end{array}$} & \multicolumn{2}{|c|}{$\begin{array}{l}\text { MODEL REGRESSION } \\
\text { (FGLS) }\end{array}$} & \multirow{2}{*}{$\begin{array}{c}\text { Kết quả kiểm định giả } \\
\text { thuyết }\end{array}$} \\
\hline & Coef. & Sta. Err. & \\
\hline $\begin{array}{l}\text { INTEREST } \\
\text { ICT }\end{array}$ & $\begin{array}{c}0.0677 * * * \\
-1.0880 * * *\end{array}$ & $\begin{array}{l}0.0174 \\
0.4880\end{array}$ & $\begin{array}{l}\text { Chấp nhận } \\
\text { Chấp nhận }\end{array}$ \\
\hline $\begin{array}{l}\text { Number of obs } \\
\text { Wald Chi2 (7) } \\
\text { AR(1) } \\
\text { Prob>chi2 }\end{array}$ & $\begin{array}{r}2 \\
14 \\
0.3 \\
0.0\end{array}$ & & \\
\hline
\end{tabular}

Mức ý nghĩa $* * * \mathrm{P}<0.01$

Nguồn: Tác giả (2017)

\subsection{Kiểm định độ phù hợp mô hình}

Kiểm định sụ phù hợp của mô hình

Giá trị kiểm định $\mathrm{F}$ cho kết quả Prob $(\mathrm{F}$-statistic $)=0.000<\alpha=0.05$ nên ta bác bỏ giả thuyết $\mathrm{H}_{0}$, chấp nhận giả thuyết $\mathrm{H}_{1}$ : mô hình nghiên cứu là phù hợp. Bên cạnh đó, $\mathrm{R}^{2}$ hiệu chỉnh của mô hình 1 bằng 0.296 . Điều này có nghĩa là với mô hình trên, các biến độc lập bên trong ngân hàng có thể giải thích được khoảng $29.6 \%$ cho sự biến thiên của NPL.

Kiểm định đa cộng tuyến

Hệ số VIF của các biến đều nhỏ hơn 5 (Bảng 5), như vậy, các biến trong mô hình không xảy ra hiện tượng đa cộng tuyến (Gujarati, 2012).

\section{Bảng 5}

Kết quả kiểm định đa cộng tuyến

\begin{tabular}{lll}
\hline \multicolumn{1}{c}{ Variable } & VIF & $\mathbf{1 / V F F}$ \\
\hline ROE & 1.34 & 0.749036 \\
LnCRE & 1.05 & 0.953160 \\
LnRISK & 1.19 & 0.840702 \\
LnASSETS & 1.08 & 0.926208 \\
AGENT_branch & 1.34 & 0.744929 \\
INTEREST & 1.12 & 0.896147 \\
ICT & 1.13 & 0.883490 \\
\hline Mean VIF & $\mathbf{1 . 1 8}$ & \\
\hline
\end{tabular}

Nguồn: Kết quả phân tích dữ liệu của nhóm nghiên cứu

$$
\text { Kiểm định phuơng sai sai số thay đổi và tụ tuơng quan }
$$

Kết quả hồi quy theo phương pháp FGLS cho thấy, Wald Chi2 (7) = 142.81 với AR(1) 
$=0.3015$ và Prob $>$ chi $2=0.000$. Như vậy, các khuyết tật về phương sai sai số thay đổi và tự tương quan của mô hình đã được khắc phục, mô hình có ước lượng không chệch, đảm bảo độ tin cậy cao.

\section{Kết luận và hàm ý chính sách}

\subsection{Kết luận}

Các yếu tố bên trong ngân hàng tác động đến tỷ lệ nợ xấu (NPL) theo phương pháp hồi quy FGLS cho thấy, có 6/7 biến độc lập có tác động, trong đó các biến ROE, LnCRE, LnASSETS và ICT có tác động trái chiều với NPL. Các biến LnRISK và INTEREST có tác động cùng chiều với NPL. Kết quả nghiên cứu phù hợp với một số nghiên cứu trước và phù hợp với thực tế tại Việt Nam. Biến AGENT branch chưa tìm thấy bằng chứng có tác động đến NPL. Dựa vào kết quả nghiên cứu cũng như tình hình thực tế tại các NHTMVN, một số khuyến nghị được đề xuất nhằm góp phần giảm nợ xấu tại các NHTMVN.

\subsection{Hàm ý chính sách}

Đối với các nhà quản trị ngân hàng: Các nhà quản trị ngân hàng có thể kiểm soát nợ xấu tại ngân hàng thông qua nhiều cách khác nhau như: gia tăng suất sinh lời ROE, trích lập dự phòng rủi ro theo đúng qui định, sử dụng hiệu quả thông tin tín dụng, thúc đẩy tăng trưởng tín dụng và tăng tài sản. Các ngân hàng có thể gia tăng ROE và sử dụng ICT càng tốt thì NPL càng giảm, trong đó, đặc biệt cần chú ý sử dụng yếu tố ICT vì hiện nay các ngân hàng tiếp cận và sử dụng hiệu quả hệ thống thông tin tín dụng chưa tốt và cũng chưa nghiên cứu sâu vấn đề này. Tuy nhiên, để hệ thống thông tin tín dụng hiệu quả, chính xác, minh bạch, ... thì cần có sự chỉ đạo, hỗ trợ của các cơ quan quản lý nhà nước, đặc biệt là Ngân hàng Nhà nước.

Đối với Ngân hàng Nhà nước: Cần gia tăng cũng cố và phát huy hệ thống thông tin tín dụng để là cơ sở hữu hiệu cho các NHTM có thể đưa ra những quyết định tín dụng kịp thời, chính xác hơn, lựa chọn đối tượng để cấp tín dụng hiệu quả, nâng cao khả năng thu hồi vốn tín dụng. Đồng thời, cơ quan quản lý Nhà nước cần nghiên cứu và đưa ra định hướng lãi suất phù hợp với nền kinh tế Việt Nam theo từng giai đoạn nhằm nâng cao khả năng hoàn trả nợ vay của người vay vốn, từ đó đảm bảo khả năng thu hồi nợ của ngân hàng.

\section{Tài liệu tham khảo}

Atlman, E. I. (2000). Predicting financial distress of companies: Revisiting the Z-Score and ZETA models. Retrieved September 1, 2017, from https://ideas.repec.org/h/elg/eechap/14545_17.html

Auronen, L. (2003). Asymmetric information: Theory and applications. Paper presented at the Seminar of Strategy and International Business, Helsinki University of Technology, Espoo, Finland.

Baltagi, B. H. (2010). Panel data econometrics, theoretical contributions and empirical application (1st ed.). Amsterdam, Netherlands: Elsevier. 
Bester, H. (1994). The role of collateral in a model of debt renegotiation. Journal Money, Credit and Banking, 26, 72-86.

Breusch, T. S., \& Pagan, A. R. (1979). A simple test for heteroscedasticity and random coefficient variation. Econometrica, 47(5), 1287-1294.

Chính phủ. (2016). Báo cáo tình hình kinh tế - xã hội 6 tháng đầu năm và các nhiệm vụ, giải pháp chủ yếu 6 tháng cuối năm 2016 [Report on socio-economic situation in the first 6 months of the year and major tasks and solutions for the last 6 months of 2016]. Retrieved September 2, 2017, from https://www.gso.gov.vn/du-lieu-va-so-lieu-thongke/2019/05/bao-cao-tinh-hinh-kinh-te-xa-hoi-6-thang-dau-nam-2016/

Chu Thai (2017). Nọ’ xấu quý I/2017: Vẫn tăng và có sự phân hóa [NPLs in the first quarter of 2017: Still increasing and having differentiation]. Retrieved September 4, 2017, from http://thoibaotaichinhvietnam.vn/pages/tien-te-bao-hiem/2017-05-17/no-Xau-quy-i2017-van tang-va-co-su-phan-hoa-43369.aspx

Dash, M., \& Kabra, G. (2010). The determinants of non-performing assets in Indian commercial bank: An econometric study, Middle Eastern Finance and Economics, 7, 94-106.

Fofack, H. (2005). Non-performing loans in sub-Saharan Africa: Causal analysis and macroeconomic implications. (World Bank Policy Research Working Paper, No. 3769). Retrieved November 15, 2005, from http://ssrn.com/ abstract=849405

Golden, S., \& Walker, H. M. (1993). The ten commandments of commercial credit. The Cs of good and bad loans. Journal of Commerial Bank Leading, 13, 42-46

Greenidge, K., \& Grosvenor, T. (2010). Forecasting non-performing loans in Barbados. Journal of Business, Finance and Economics in Emerging Economies, 5(1), 80-107.

Gujarati, D. N. (2004). Basic econometric (4th ed.). NewYork, NY: The McGraw-Hill Companies.

Gujarati, D. N. (2012). Basic econometrics. New York, NY: Tata McGraw-Hill Education.

Guy, K., \& Lowe, S. (2011). Non-performing loans and bank stability in Barbados. Economic Review, 37(1), 77-82.

Harper, B. (2011). Linking banks and strong economic growth, ABA occasional paper. Retrieved September 8, 2017, from https://www.coursehero.com/file/45550217/1Group-13-Importance-of-bankspdf/

International Monetary Fund (IMF). (2004). Compilation guide on Financial Soundness Indicators-FSIs. Retrieved September 9, 2017, from https://www.imf.org/ external/np/sta/fsi/eng/2004/guide/index.htm.

Jiménez, G., \& Saurina, J. (2006). Credit cycles, credit risk, and prudential regulation. International Journal of Central Banking, 2(2), 65-98.

Louzis, D. P., Vouldis, A. T., \& Metaxas, V. (2010). Macroeconomic and bank-specific determinants of non-performing loans in Greece: A comparative study of mortgage, 
business and consumer loan portfolios. (Bank of Greece Working Paper No. 118/2010). Retrieved September 11, 2017, from http:ssrn.com/abstract=1703026

Louzis, D. P., Vouldis, A., \& Metaxas, V. (2012). Macroeconomic and bank-specific determinants of non- performing loans in Greece: A comparative study of mortgage, business and consumer loan portfolios. Journal of Banking \& Finance, 36(4), 1012-1027.

Mario, Q. (2006). Bank's riskiness over the business cycle: A panel analysis on Intalian intermediaries. (Bank of Italy Working Papers, No. 559). Retrieved September 11, 2017, from http://ssrn.com/abstract $=935021$

Messai, A. S., \& Jouni, F. (2013). Micro and macro determinants of non-performing loans. International Journal of Economic and Financial Issues, 3(4), 852-860.

Ngân hàng Nhà nước Việt Nam. (2005). Quyết định số 493/2005/QĐ-NHNN ban hành ngày 22/04/2005 [Decision No. 493/2005 / QD-NHNN dated 22/04/2005]. Retrieved September 12, 2017, from https://thuvienphapluat.vn/van-ban/Tien-te-Nganhang/Quyet-dinh-493-2005-QD-NHNN-phan-loai-no-trich-lap-su-dung-du-phong-dexu-ly-rui-ro-tin-dung-trong-hoat-dong-ngan-hang-to-chuc-tin-dung-53338.aspx

Ngân hàng Nhà nước Việt Nam. (2013). Thông tu số 02/2013/TT-NHNN ban hành ngày 21/01/2013 [Circular No. 02/2013 / TT-NHNN dated 21/01/2013]. Retrieved September 13, 2017, from https://thuvienphapluat.vn/van-ban/Tien-te-Ngan-hang/Thong-tu-022013-TT-NHNN-phan-loai-tai-san-co-muc-trich-phuong-phap-trich-lap-165814.aspx

Nguyen, K. M. (2015). Giáo trình Nghiệp vu ngân hàng thương mại [Commercial Banking Textbook]. Ho Chi Minh, Vietnam: Nxb Tài chính.

Nguyen, V. T. H. (2015). Yếu tố tác động đến nợ xấu các ngân hàng thương mại Việt Nam [Factors affecting bad debts of commercial banks in Vietnam]. Tạp chí Phát triển Kinh tế, 26(11), 80-98.

Pham, B. T. (2005). Nâng cao năng lục cạh tranh của hệ thống NHTM Việt Nam trong điều kiện họi nhập khu vưc và quốc tế [Improve the competitiveness of Vietnam's commercial banking system in terms of regional and international integration] (Report No. KNHTĐ 2003.01).

Podpiera, J., \& Weill, L. (2008). Bad luck or bad management? Emerging banking market experience. Journal of Financial Stability, 4(2), 135-148.

Rajan, R., \& Dhal, S. C. (2003). Non-performing loans and terms of credit of public sector banks in India: An empirical assessment. Proceeding of Reserve Bank of India Occasional Paper, 24, 81-121.

Richard, E. (2011). Factors that cause non-performing loans in commercial banks in tanzania and strategies to resolve them. Journal of Management Policy and Practice, 12(7), 5058 .

Salas, V., \& Saurina, J. (2002). Credit risk in two institutional regimes: Spanish commercial and savings banks. Journal of Financial Services Research, 22(3), 203-224. 
Sinkey, J. F., \& Greenwalt, M. (1991). Loan-loss experience and risk-taking behavior at large commercial banks. Journal of Financial Services Research, 5(1), 43-59.

Tran, H. H. (2011). Giáo trình quản trị ngân hàng thuoơng mại [Commercial banking management curriculum]. Hanoi, Vietnam: Nxb Lao động Xã hội.

Upal, R. (2009). Priority sector advances: Trends, issues and strategies. Journal of Accounting and Taxation, 1(5), 79-89.

Wooldridge, J. M. (2002). Introductory econometrics - A modern approach. New York, NY: McGraw-Hill. 